\title{
The prevalence, risk factors and outcomes of anaemia in South African pregnant women: a protocol for a systematic review and meta-analysis
}

\author{
Vinogrin Dorsamy ${ }^{1 *}$ (D) Chauntelle Bagwandeen ${ }^{2}$ and Jagadesa Moodley ${ }^{3}$
}

\begin{abstract}
Background: A significant cause of morbidity and mortality during pregnancy is maternal anaemia. The causes of anaemia and the sequelae are varied, and the prevention and management are public health challenges, especially in resource-limited settings and certain geographic locations. South Africa is plagued by a quadruple burden of disease, with high maternal mortality rates affected by hypertensive disorders of pregnancy, HIV, tuberculosis and neglected tropical diseases. This is most prevalent in people of lower socio-economic status. Poor nutrition, chronic infections, lack of access to health care facilities and poor compliance with micronutrient supplementation all contribute to maternal anaemia. The aim of this study is to systematically map the literature to ascertain the pooled prevalence and associated causes of anaemia in the South African pregnant population, which will enable health care workers and other key stakeholders to more pertinently address Sustainable Development Goal 3 focussing on good health and reducing maternal mortality.

(Continued on next page)
\end{abstract}

\footnotetext{
* Correspondence: dorsamyv1@ukzn.ac.za

'Laboratory Medicine and Medical Sciences, College of Health Sciences, University of KwaZulu-Natal, KwaZulu-Natal, South Africa

Full list of author information is available at the end of the article
}

C C The Author(s). 2020 Open Access This article is licensed under a Creative Commons Attribution 4.0 International License, which permits use, sharing, adaptation, distribution and reproduction in any medium or format, as long as you give appropriate credit to the original author(s) and the source, provide a link to the Creative Commons licence, and indicate if changes were made. The images or other third party material in this article are included in the article's Creative Commons licence, unless indicated otherwise in a credit line to the material. If material is not included in the article's Creative Commons licence and your intended use is not permitted by statutory regulation or exceeds the permitted use, you will need to obtain permission directly from the copyright holder. To view a copy of this licence, visit http://creativecommons.org/licenses/by/4.0/. The Creative Commons Public Domain Dedication waiver (http://creativecommons.org/publicdomain/zero/1.0/) applies to the data made available in this article, unless otherwise stated in a credit line to the data. 
(Continued from previous page)

Methods: PubMed, CINAHL, EMBASE, EBSCO, Ovid maternity and infant care databases, Cochrane Database of Systematic Reviews, Web of Science and SCOPUS will be searched using the keywords 'anaemia', 'haemoglobin', 'pregnancy', and 'South Africa' to conduct a systematic review and meta-analysis to explore, describe and map literature on the distribution and burden of anaemia in pregnant women in South Africa. The reference list of articles selected for review will be scanned for other articles of interest to our study question. Studies published in any language will be included in this review. As there may be differences in sampled populations in South Africa based on geography and sociodemographic factors, a weighted inverse-variance meta-analysis using a random-effects model will be carried out to generate a pooled prevalence estimate. A Funnel plot and Egger's regression test will be conducted to assess publication bias. Heterogeneity among studies will be checked using $P^{2}$ to determine dispersion and meta-regression analysis will be performed to investigate the source of heterogeneity. The articles obtained by these searches will be analysed for causative factors, severity and outcomes by a parallel and independent review team, using suitable eligibility criteria. Screening, data extraction and quality appraisal will be conducted independently by two authors. Disagreement will be resolved by independent assessment by a third reviewer. Sub group analysis by region, stage of pregnancy, socio-economic status, severity and cause of anaemia will be conducted if sufficient data is available. Data will be analysed using statistical software and presented in evidence tables and in meta-analytic forest plots.

Discussion: This protocol is developed to systematically review the literature on the prevalence and severity of anaemia, risk factors and outcomes in pregnant women in South Africa. Correlation of factors contributing to the development of anaemia and other disorders during pregnancy will facilitate exploration of appropriate medical and behavioural change interventions implemented within other countries or regions that mitigate risk. This study will assist local health systems to inform public health policies and practises for more favourable maternal and fetal outcomes.

Trial registration: This protocol is registered with PROSPERO (CRD42020157191)

Keywords: Anaemia, Pregnancy, South Africa

\section{Background}

Anaemia, defined as a decrease in red blood cells or haemoglobin, or the reduced capacity of red blood cells to carry oxygen [1] is a common chronic problem in low- to middle-income countries, such as South Africa. It is a product of socio-economic disparity, affecting mostly the poor and less educated [2]. It is most prevalent in the vulnerable: children, adolescents in their growth phase and females of reproductive age [3]. The condition is exacerbated by pregnancy when there is an increased burden to deliver oxygen to the fetus and can result in intrauterine growth restriction, reduced iron for the baby and increased maternal and perinatal morbidity and mortality [4].

Anaemia affects almost 2 billion people worldwide, with iron deficiency anaemia being the commonest cause. As with other low- and middle-income countries with similar sociodemographic characteristics, the South African population is more susceptible to anaemia due to the prevalence of chronic nutritional insufficiency and concomitant infective conditions [5]. The World Health Organisation (WHO) reports the prevalence of anaemia in women of reproductive age women in sub-Saharan Africa to be $57.1 \%$ [6], but this varies between regions, with the highest in Central and West Africa (61\%) and the lowest level reported in Southern Africa (34\%) in 2011. While the authors report a declining trend from 1995 to 2011, current prevalence data for the region is not available, and data for anaemia in pregnant South African women is outdated. The last census type study was conducted in 2012, and more current studies report on isolated sites only.

Food fortification and supplements during gestation are the mainstay of prophylaxis against the sequelae of iron deficiency [7]. However, anaemia is also due to other causes. In South Africa, pregnant women face the compound burden of infective causes of anaemia, such as HIV and TB, neglected tropical diseases, like hookworm and schistosomiasis, other bacterial infections, as well as nutritional insufficiency and inherited haemoglobinopathies and thalassaemias, which may also exacerbate negative pregnancy outcomes [6].

However, given that physiological anaemia in pregnancy is associated with protection against fulminant expression of infections such as schistosomiasis and other parasitaemias, non-discriminatory mass treatment with iron supplementation in these cases may contribute adversely to maternal and fetal morbidity and mortality [8].

It is therefore important to determine the current burden, causes and consequences of anaemia in pregnant women so as to better guide appropriate treatment protocols which will focus on the specific cause of anaemia and may differ to current recommendations.

\section{Aim and objectives}

It is the purpose of this systematic review to establish the present pooled prevalence of anaemia in South African pregnant women. The specific objectives are to 
- Determine the causal or associated factors of anaemia in the pregnant population

- Describe the type and severity of anaemia

- Describe the fetal and maternal outcomes associated with anaemia in pregnancy

Correlation of factors contributing to the development of anaemia and other disorders during pregnancy will facilitate exploration of medical interventions as well as inform behavioural changes to mitigate current and future risk.

\section{Methodology}

This protocol is written in line with the recommendations of the Preferred Reporting Items for Systematic Reviews and Meta-analysis for Protocols (PRISMA-P) guidelines [9]. The results will be reported based on the PRISMA 2009 statement, and article screening and selection process will also be demonstrated through a PRISMA-P flow diagram.

\section{Eligibility of research question}

The eligibility of the research question was determined by the Population Exposure Comparator Outcomes (PECO) framework as illustrated in Table 1.

All studies reporting on anaemia and reporting on prevalence or haemoglobin concentration during pregnancy in a South African population will be included. There will be no restriction on study design, setting or date of publication. Articles published in any language will be accepted and translated into English using Google Translate if possible. If it is not possible to translate abstracts into English, they will be removed from the study, but listed in an appendix.

\section{Identifying relevant studies}

Search terms used and their synonyms were identified using the Medical Subject Headings (MeSH). The uniterms and Boolean operators in English to be used in the search strategies are (anemia OR anaemia OR haemoglobin OR hemoglobin OR haematocrit OR hematocrit) AND (Pregnancy OR Pregnant women OR Gravidity OR Maternal exposure OR Mother OR Pregnant OR Gravid OR Obstetric OR Antenatal OR Antepartum OR Gestation) AND (South Africa OR Southern Africa or South African or Sub-Saharan Africa). The

\begin{tabular}{ll} 
Table 1 PECO framework for eligibility of research question \\
\hline $\begin{array}{l}\text { Population } \\
\text { Intervention/ } \\
\text { exposure }\end{array}$ & $\begin{array}{l}\text { Pregnant women with anaemia in South Africa } \\
\text { Comparator }\end{array}$ \\
Outcomes & $\begin{array}{l}\text { Pregnant women without anaemia in any trimester } \\
\text { Prevalence, severity, risk factors, maternal morbidity }\end{array}$ \\
& $\begin{array}{l}\text { and mortality, fetal outcomes } \\
\end{array}$
\end{tabular}

search strategy will be adapted for the other electronic databases to be searched.

The search strategy was piloted in November 2019 to check the appropriateness of selected electronic databases and key words as depicted in Table 2 .

\section{Study selection}

The eligibility of retrieved studies will be assessed using the following criteria:

Inclusion criteria

For studies to be included they should meet the following criteria:

- Be available in full text

- Must include pregnant women living in South Africa

- Must focus on anaemia in pregnancy using the WHO classification of anaemia: $<10 \mathrm{mg} / \mathrm{dL} \mathrm{Hb}$ or include such data on pregnant women in studies ascertaining prevalence in various population groups

Exclusion criteria

Studies will be excluded should they be:

- Not available in full text

- Not reporting anaemia or haemoglobin concentrations in pregnancy

- Full papers not available despite requests to the authors

An abstract screening tool, using Google Forms, will be developed and distributed to the researchers. Abstract screening, followed by full article screening, will be conducted manually, including those articles for which an abstract is not available.

A citation manager Zotero v5.0.81 (Zotero.org) will be used to create a library for this review. The primary investigator (VD) will conduct a search using the key fields in the databases created. Eligible studies will be exported to the citation manager, with duplicates removed before abstracts are screened by two authors/investigators (VD and $\mathrm{CB}$ ). A test of interrater reliability using Cohen's Kappa will be performed with a cut-off point of .80 (indicating substantial agreement). If this precision is not

\begin{tabular}{|c|c|c|}
\hline $\begin{array}{l}\text { Database } \\
\text { used }\end{array}$ & Search terms & $\begin{array}{l}\text { Articles } \\
\text { found }\end{array}$ \\
\hline PubMed & $\begin{array}{l}\text { (((((((("anaemia") OR "anemia") OR "haemoglobin") } \\
\text { OR "hemoglobin") OR "iron deficiency") OR } \\
\text { "haematocrit") OR "hematocrit")) AND } \\
\left(\left(\left(\left(\left(\left(\left(\left(\left(\left({ }^{\prime} \text { pregnancy") OR "pregnant") OR }\right.\right.\right.\right.\right.\right.\right.\right.\right.\right. \\
\text { "maternal") OR "mother") OR "gravid") OR } \\
\text { "maternal exposure") OR "obstetric") OR } \\
\text { "antenatal") OR "antepartum") OR "gestation") OR } \\
\text { "gestational")) AND ((("south africa") OR "southern } \\
\text { africa") OR "south african" OR "Sub-Saharan") }\end{array}$ & 486 \\
\hline
\end{tabular}


met, all discrepancies in selected abstracts will be evaluated by the third author/investigator (JM) for eligibility. Full article screening guided by the eligibility criteria will then be carried out independently. A subject librarian will be consulted to optimise the article search procedure to help with retrieving and finding articles to be included in the fullarticle screening. For those papers that are electronically unavailable, authors will also be contacted directly. The screening results will be reported using a PRISMA-P flowchart [9].

\section{Charting of data items}

As illustrated in Table 3 below, a data charting table will be used to extract background information and process the data items from each study selected. To ensure that all pertinent information regarding the relevant aspects for the study is collected, a data charting form will be developed and piloted, and continually updated.

\section{Collating, summarising and reporting the results Risk of bias and quality assessment}

The quality and risk of bias of selected studies will be performed by both reviewers using the Hoy tool [10] which determines the risk of bias in prevalence studies. The tool uses 10 criteria to assess both internal and external validity of studies based on participant selection criteria as a representation of overall population, how information is collected, and whether parameters used to determine prevalence was consistent in each study amongst participants [10]. An overall risk of bias will then be determined based on the individual scores obtained for each of the 10 items and graded according to the number of high risks found: low $(\leq 2)$, moderate $(3,4)$ and high $(\geq 5)$. Where information related to the 10 items is not available, it will be captured as high risk.

The quality and scientific evidence of the studies will be determined using the Grading of Recommendations Assessment, Development and Evaluation (GRADE) tool to assess risk of bias, imprecision, inconsistency and indirectness, as well as publication bias, and placed into one of four categories, ranging from very low (where the true effect is probably markedly different from the estimated effect) to very high (that which was observed is most likely to be true). Randomised controlled trials without limitations will therefore present the most reliable evidence, with observational studies falling into the lowest category, given the potential for confounding. The strength of the recommendations emanating from these studies will be assessed based on the quality of evidence presented. The two authors (VD and $\mathrm{CB}$ ) will independently assess the quality of the studies using the data extraction tool, with consensus on disagreement being achieved with the assistance of a third author (JM).

\section{Data synthesis and analysis}

Heterogeneity among studies will be checked using $I^{2}$ to determine dispersion. As there may be substantial heterogeneity
Table 3 Data charting table

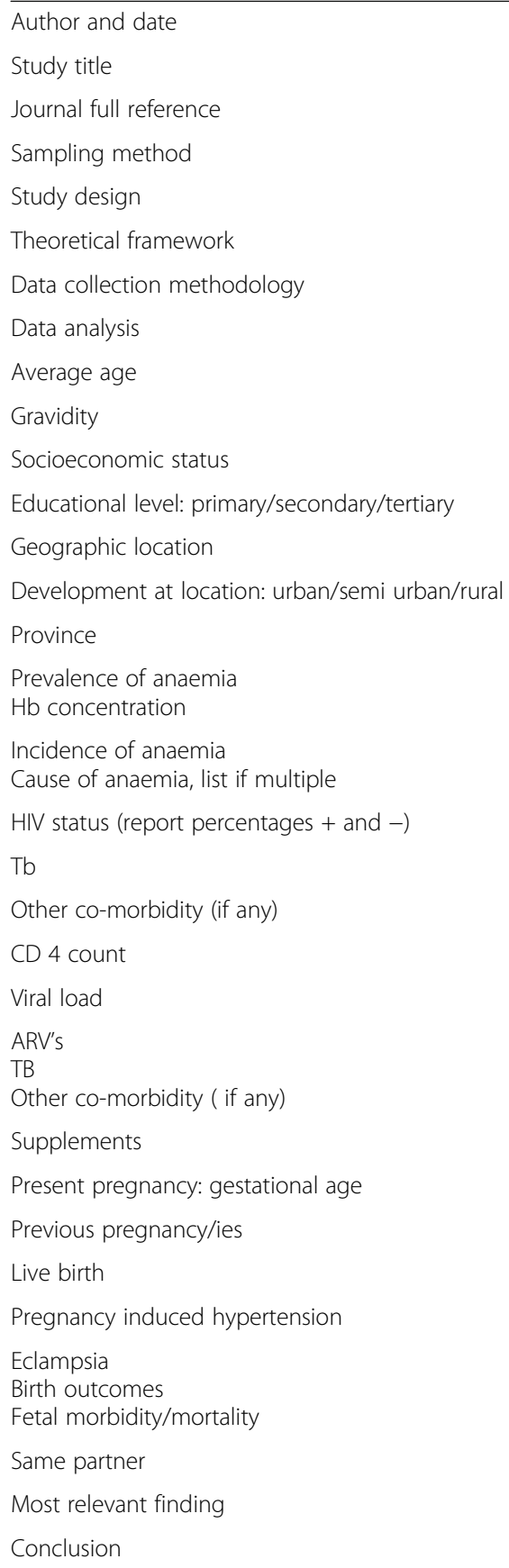

based on differences in sampled populations in South Africa based on geography and sociodemographic factors, a weighted inverse-variance meta-analysis using a randomeffects model will be carried out to generate a pooled prevalence estimate. A Funnel plot and Egger's regression test will be conducted to assess publication bias. The articles obtained by these searches will be analysed for causative factors and outcomes by a parallel and independent review team, using suitable eligibility criteria. Screening, data extraction, and 
quality appraisal will be conducted independently by two authors (VD and $\mathrm{CB}$ ). Disagreement will be resolved by independent assessment by a third reviewer (JM). Sub group analysis by region, stage of pregnancy, socio-economic status, severity, and cause of anaemia will be conducted if sufficient data is available. Data will be analysed using STATA v16 (2019, College Station, TX: StataCorp. LLC.) and presented in evidence tables and in meta-analytic forest plots.

\section{Discussion}

Thus far very few investigations have focussed on anaemia and concomitant disorders during pregnancy in the Southern African region. Active research in this vulnerable population is urgently required to identify the type and burden of anaemia affecting South African pregnant women and its association with contributing factors such as nutrient deficiency, growth and development of the fetus and neonate, co-morbid diseases such as TB and HIV, hypertensive disorders of pregnancy as well as clinical outcomes and longterm sequelae. This protocol has been developed to determine the prevalence, type and causes of anaemia affecting pregnant women in South Africa. The information thus obtained will best inform decision-makers in developing health policies and practises most suitable to positively impact on maternal and fetal outcomes.

\section{Supplementary information}

Supplementary information accompanies this paper at https://doi.org/10. 1186/s13643-020-01460-0.

Additional file 1. Prisma $P$ Checklist

\section{Abbreviations}

WHO: World Health Organisation; HIV: Human immunodeficiency virus; TB: Tuberculosis; PRISMA-P: Preferred Reporting Items for Systematic Reviews and Meta-analysis for Protocols; PECO: Population Exposure Comparator Outcomes; Hb: Haemoglobin; GRADE: Grading of Recommendations Assessment, Development and Evaluation

\section{Acknowledgements}

The authors would like to thank Dr. Tivani Mashamba-Thomson and Dr. Bongani Nkambule for their guidance in the development of this protocol.

\section{Amendments}

This is the first iteration of the protocol. Should it be necessary to amend any part of this protocol, every amendment including the date, the nature of the change and the rationale behind it will be registered independently of the protocol.

\section{Authors' contributions}

VD and CB conceptualised the study and prepared the draft protocol under the guidance and supervision of JM. All three authors contributed to the development of the background and planned output of the research as well as the design of the study. All authors read and approved the final manuscript.

\section{Funding}

This protocol for a systematic review forms part of a larger study investigating anaemia and hypertensive disorders of pregnancy in South African women, which is funded by the National Research Foundation Thuthuka Grant (TTK170508230162) in collaboration with the University of KwaZulu-Natal and Medical Research Council of South Africa (SIR Grant UNS14197). The institutions listed have no vested interest in the study and are not involved in the protocol design, analysis plan, data collection and analyses. Further, there will be no input from these organisations in the interpretation and publication of the results.

Availability of data and materials

All data generated or analysed during this study will be included in the published systematic review article and will also be available upon request.

The Prisma-P checklist is available in Additional file 1.docx

Ethics approval and consent to participate

Not applicable.

\section{Consent for publication}

Not applicable.

\section{Competing interests}

The authors declare that they have no competing interests.

\section{Author details}

${ }^{1}$ Laboratory Medicine and Medical Sciences, College of Health Sciences, University of KwaZulu-Natal, KwaZulu-Natal, South Africa. ${ }^{2}$ Discipline of Public Health Medicine, College of Health Sciences, University of KwaZulu-Natal, KwaZulu-Natal, South Africa. ${ }^{3}$ Women's Health and HIV, School of Clinical Medicine, University of KwaZulu-Natal, KwaZulu-Natal, South Africa.

Received: 29 February 2020 Accepted: 21 August 2020

Published online: 11 September 2020

\section{References}

1. Younger P. Stedman's Medical Dictionary, 28th ed.200734 Stedman's Medical Dictionary, 28th ed. . Philadelphia, PA: Lippincott Williams and Wilkins 2006. I+2170 pp.+appendices 176 pp. £26.95; \$49.95, ISBN: 0781733901 Includes CD-ROM. Ref Rev. 2007:21(1):41-2.

2. Shisana O. South Africa, Department of Health, Human Sciences Research Council, South African Medical Research Council. The South African National Health and Nutrition Examination Survey: SANHANES-1. Cape Town: HSRC Press; 2013.

3. Stevens FMM, De-Regil LM, Paciorek CJ, Flaxman SR, Branca F, et al. Global, regional, and national trends in haemoglobin concentration and prevalence of total and severe anaemia in children and pregnant and non-pregnant women for 1995-2011: a systematic analysis of population-representative data. Lancet Glob Health. 2013;1(1):e16-25.

4. Hoque M. Risk factors for anaemia in pregnancy in rural KwaZulu-Natal, South Africa : implication for health education and health promotion : original research. 2009 [cited 2015 Nov 14]; Available from: http://reference. sabinet.co.za/document/EJC80328.

5. Mkhize BT, Mabaso M, Mamba T, Napier CE, Mkhize-Kwitshana ZL. The Interaction between HIV and Intestinal Helminth Parasites Coinfection with Nutrition among Adults in KwaZulu-Natal. South Africa. BioMed Res Int. 2017 Mar 22;2017:e9059523.

6. Soares Magalhães RJ, Clements AC. Spatial heterogeneity of haemoglobin concentration in preschool-age children in sub-Saharan Africa. Bull World Health Organ. 2011;89(6):459-68.

7. Cantor AG, Bougatsos C, Dana T, Blazina I, McDonagh M. Routine iron supplementation and screening for iron deficiency anemia in pregnancy: a systematic review for the U.S. Preventive Services Task Force. Ann Intern Med. 2015 Apr 21;162(8):566-76.

8. Haider BA, Olofin I, Wang M, Spiegelman D, Ezzati M, Fawzi WW, et al. Anaemia, prenatal iron use, and risk of adverse pregnancy outcomes: systematic review and meta-analysis. BMJ. 2013 Jun 21:346:f3443.

9. Moher D, Shamseer L, Clarke M, Ghersi D, Liberati A, Petticrew M, et al. Preferred reporting items for systematic review and meta-analysis protocols (PRISMA-P) 2015 statement. Syst Rev. 2015;4(1):1

10. Hoy D, Brooks P, Woolf A, Blyth F, March L, Bain C, et al. Assessing risk of bias in prevalence studies: modification of an existing tool and evidence of interrater agreement. J Clin Epidemiol. 2012 Sep;65(9):934-9.

\section{Publisher's Note}

Springer Nature remains neutral with regard to jurisdictional claims in published maps and institutional affiliations. 